\title{
Dynamic consistency of expected utility under non-classical (quantum) uncertainty
}

\author{
Danilov V.I.*, Lambert-Mogiliansky A.†, and V. Vergopoulos ${ }^{\ddagger}$
}

October 6, 2018

\begin{abstract}
Quantum cognition in decision-making is a recent and rapidely growing field. In this paper we develop an expected utility theory in a context of non-classical (quantum) uncertainty. We replace the classical state space with a Hilbert space which allows introducing the concept of quantum lottery. Within that framework we formulate axioms on preferences over quantum lotteries to establish a representation theorem. We show that demanding the consistency of choice behavior conditional on new information is equivalent to the von Neuman-Lüders postulate applied to beliefs. A dynamically consistent quantum-like agent may violate dynamic recursive consistency, however. This feature suggests interesting applications in behavioral economics as we illustrate in an example of persuasion.
\end{abstract}

\section{Introduction}

Alternatives available in decision problems can often be analyzed in terms of a variety of perspectives: a fur coat may be evaluated from an esthetical point of view or from the point of view of animal suffering. A military intervention in Syria can be evaluated from a geopolitical perspective or from a humanitarian one. Another type of example relates to the

\footnotetext{
*Central Mathematic Economic Institute, Russian Academy of Sciences, vdanilov43@mail.ru.

${ }^{\dagger}$ Paris Scool of Economics, alambert@pse.ens.fr

†Université Paris 1 Panthéon-Sorbonne and Paris School of Economics, vassili.vergopoulos@univ-paris1.fr
} 
consumption of cigarettes: the immediate pleasure perspective contra the long term health perspective. In order to assess an alternative we need to build a representation of it, a "represented alternative" which is a mental construct11. Standard decision theory postulates that we always are able to combine any relevant perspectives into a synthetic and stable representation of the alternatives. However, it is also a common place for cognitive scientists that we face difficulties when building our representation of a complex alternative. We consider the alternative from different perspectives - one at a time. And most importantly we are not always able to synthesize information from various perspectives into one single coherent and stable representation of the alternative.

In this paper we are interested in decision-making under uncertainty and we want to capture the difficulties people show in combining all relevant information by analogy with incompatible properties in Quantum Mechanics. To many people it may appear unmotivated or artificial to turn to quantum mechanics (QM) when investigating human behavioral phenomena. However, the founders of QM, including Bohr and Heisenberg, were early to recognize an essential similarity between the two fields:2 in both fields the object of investigation cannot (always) be separated from the process of investigation. QM and in particular its mathematical formalism was developed to respond to a general epistemological challenge: how can one study an object that is being modified by the measurement of its properties? This provides legitimacy to the exploration of the value of the mathematical formalism of QM in the study of human behavioral phenomena without reference to Physics 3 Of particular interest in our context is that this formalism allows representing agents subject to the incapacity to simultaneously consider a choice alternative from all relevant perspectives. For instance when evaluating the "animal suffering" value of a fur coat, its esthetical (subjective) value, that was well-determined in our decision-maker's mind before considering animal suffering aspects, may become "blurred" i.e., uncertain.

The classical approach to decision-making under uncertainty e.g., in Savage (1972) and

\footnotetext{
${ }^{1}$ Kahneman and Tversky (2000) write "the true objects of evaluation are neither objects in the real world nor verbal descriptions of those objects; they are mental representations" a conception which they further write is entirely natural for cognitive scientists (p. xiv).

${ }^{2}$ In particular Bohr was influenced by the psychology and philosophy of knowledge of Harald Höffding (see Bohr 1971 and the Introduction in Bitbol 2009 for an insightful discussion).

${ }^{3}$ The human mind behaves in a wide array of weird manners. It is not the weirdness of quantum mechanics that makes it an attractive toolbox, but the fact that it is a most general paradigm for structural contextuality (i.e., non-separability between the object of and the operation of investigation).
} 
Anscombe and Aumann (1963) builds on the notion of a state space $S$ of states of nature. Very roughly a representation of the world (a belief) corresponds to a probability distribution on $S$. And the changes in beliefs following the acquisition of new information follow Bayes' rule which can be given a behavioral foundation as shown in Ghirardato (2002) : Bayesian updating secures dynamic consistency i.e., it secures that choices based on updated preferences are consistent with ex-ante preferences defined for the condition (event) that triggered updating. There exists however massive evidence of violations of Bayes rule. One source of violations is that measurements (in a broad sense) affect the object of measurement. Most clearly this happens in quantum physics and it is the reason why some properties may be incompatible. This is formally expressed in the non-commutativity of measurement operations which induces a non-Bayesian updating process. A related line of motivation appeals to the growing interest for applications of elements of the mathematical formalism of Quantum Mechanics to psychology, social sciences and in particular to decision-making (see e.g., Brandenburger and La Mura (2015), Khrennikov (2014) and Busemeyer and Bruza (2012) for an overview of the field). The approach has shown successful in explaining a large variety of behavioral anomalies in decision-making ranging from cognitive dissonance, preference reversal, conjunction fallacy, disjunction effects to framing effects.

In a recent book Akerlof and Schiller (2015) labelled a new term "Phishing equilibrium" to express how markets systematically exploits the manipulability of real consumers with far reaching implications for the efficiency and welfare properties of free markets. In a last section we suggest in a simple economic example that quantum indeterminacy of beliefs implies a "manipulability" of economic agents much in line with Akerlof and Schiller's empirical evidence as well as with their understanding of the underlying psychological mechanism "Just change people's focus and one changes the decisions they make" (p.173).

In this article, we substitute the Boolean lattice of events with a more general lattice of projectors in the Hilbert space as the suitable framework for modelling decision-making. The notions are introduced progressively and require no previous knowledge of Quantum Mechanics or Hilbert spaces. We show that a natural definition of a quantum lottery allows for the formulation of decision theoretical axioms similar to the classical ones with one exception. We need axiom A0 that secures the stability of preferences over lotteries defined 
over different perspectives (resolutions of the state space). This axiom (that we labelled "noframing") is trivially satisfied in the classical world (all lotteries can be expressed in a single finest partition(resolution) of the state space). We next show that the von Neumann-Lüders projection postulate of Quantum Mechanics used as an updating rule is both necessary and sufficient for dynamic consistency of preferences. In our context the von NeumannLüders postulate arises from purely behavioral considerations that is from a requirement of consistency applying to conditional (on new information) preference relations. Interestingly, the specificity of non-classical uncertainty (also referred to as "contextuality") is shown to imply a failure of the so-called "recursive dynamic consistency" (a dynamic version of the Savage's Sure Thing Principle). We use this result to show that quantum indeterminacy of beliefs implies a significant "manipulability" of consumers.

There exists a few earlier works addressing quantum probabilities in the context of decision-making. These include Deutsch (1999), Pitowsky (2003), Lehrer and Shmaya (2006), Danilov and Lambert-Mogiliansky (2010) and Gyntelberg and Hansen (2012)). In particular Pitowsky writes about "betting on quantum measurements" but he is not working with preference relations. Interestingly, he formulates a rule saying that the probability for any specific outcome is independent of the specific measurement that yields it as one of its possible results. This rule is very much in line with our axiom A0. Lehrer and Shmaya propose a subjective approach to quantum probabilities but they do not work with quantum lotteries. Danilov and Lambert-Mogiliansky develop an expected utility theory in a general non-classical uncertainty context (ortho-modular lattices). A first distinction with the present work is that instead of assuming the existence of a certainty equivalent, we build on fundamentals which brings us closer to the approach of von Neumann and Morgenstein and Aumann. We also adopt the structure of the Hilbert space which allows addressing more general type of lotteries 4 These steps are necessary to develop the core contribution of the paper which is related to the dynamics of beliefs and choices in response to new information in a non-classical uncertainty environment. Gyltenberg and Hansen (2012) work with Hilbert space to develop an expected utility theory with subjective events. Their static setting shows

\footnotetext{
${ }^{4}$ In Danilov and Lambert-Mogiliansky 2010, only direct measurement (orthogonal resolution of the unit) were considered. In the present work we also address "fuzzy" measurement by means of POVM (positive operator valued measurements). In such a context an outcome is a probability distribution over events.
} 
similarities with ours. However their analysis appeals to a large number of axioms - 12 where we have 5 - and most importantly they do not address the issue of dynamic consistency.

The present work is a contribution to both decision theory and the foundations of quantum cognition. We extend previous works in two directions. First, we provide a complete characterization of expected utility theory under non-classical (quantum) uncertainty: a concise formulation of sufficient and necessary axioms in terms of preferences over quantum lotteries. Most importantly, this construction allows for a transparent characterization of dynamic consistency of choice behavior in such an environment. Finally, we discuss the value of the approach for economics and illustrate it with an example of "Phishing for Phools".

The paper proceeds as follows. First, we introduce the concept of quantum lottery which gives the opportunity to define basic elements of the mathematical formalism. In section 3 we provide a straightforward construction and a complete characterization of preferences over quantum lotteries satisfying some standard properties. We formulate the corresponding axioms and derive our representation theorem. In section 4 we address the issue of information updating and formulate our central theorem of dynamic consistency. Thereafter we discuss the value of our results in economics and end with some concluding remarks.

\section{Quantum lotteries}

We are interested in a decision-maker's preferences over what we call quantum lotteries. In this section we define the notion of quantum or Q-lottery. As for any lottery, the prize that the DM obtains depends on the realization of some event which is the outcome of a measurement, it is an uncertain payoff. And the lotteries described below (roulette, horse and quantum lotteries) differ essentially in the type of measurement that is being performed. Therefore we first need to clarify the meaning of measurement and in particular of a quantum measurement. But we shall start by reminding basic facts about roulette lotteries and so called 'horse lotteries'.

\section{Roulette lotteries}

Hereafter we let $X$ denote a set of prizes. A roulette lottery (with prizes in $X$ ) is defined by a collection of prizes $x_{1}, \ldots, x_{r}$ together with the probabilities $p_{1}, \ldots, p_{r}$ ( $p_{i}$ are non-negative real numbers with $\sum_{i} p_{i}=1$ ) for obtaining the corresponding prize. Such a lottery can 
be written as the string $l=\left(x_{1}, p_{1} ; \ldots ; x_{r}, p_{r}\right)$, but we prefer to write it as a formal sum $l=\sum_{i} x_{i} \otimes p_{i}$. We could think of it in the following way: a measurement in the form of a 'roulette' is performed and gives an outcome in the set $\{1, \ldots, r\}$. The probability of outcome $i$ is $p_{i}$ and, depending on the outcome of this 'measurement', a prize $x_{i}$ is paid.

Such lotteries can be identified with (simple) probabilistic measures on the set $X$. We denote by $\Delta(X)$ the set of such measures (or lotteries). Under well-known conditions, von Neumann and Morgenstern obtained that the utility of a lottery $l=\sum_{i} x_{i} \otimes p_{i}$ for the decision-maker DM is given by a number $U(l)=\sum_{i} p_{i} u\left(x_{i}\right)$. Here $u: X \rightarrow \mathbb{R}$ is a 'utility function' defined on the set $X$ of prizes.

\section{Horse lotteries}

The next concept is that of a 'horse lottery' (in the terminology of Anscombe and Aumann) or 'act' (in Savage's terminology). A horse lottery is a mapping $f: S \rightarrow X$ from the set $S$ of 'states of nature' to the set $X$ of prizes. A measurement is performed in the form of a 'horse race' and, depending on the result of this measurement, the corresponding prize is paid.

Again under suitable conditions the utility of a horse lottery $f$ can be written as $U(f)=$ $\sum_{s} p_{s} u(f(s))$, where $u: X \rightarrow \mathbb{R}$ is again a utility function, and $p$ is a (subjective) probability measure on the set $S$. A considerable simplification of the conditions was achieved by Anscombe and Aumann when taking roulette lotteries as prizes. They define a horse lottery as a function $L: S \rightarrow \Delta(X)$. A measurement defines the state $s$ of nature, after that a drawing of the lottery $L(s)$ performs which gives a resulting prize.

In order to smoothly move over to quantum lotteries, it is convenient to present horse lotteries slightly differently. We denote by $l(s, x)$ the corresponding probabilities for realization of outcomes $x$ in the lottery $L(s)$. Now we can form the functions $L_{x}: S \rightarrow \mathbb{R}$ by the rule $L_{x}(s)=l(s, x)$. And rewrite our horse lottery $L$ as $\sum_{x} x \otimes L_{x}$. Here the function $L_{x}$ can be understood as a plausibility (or as a potentiality) of getting prize $x$.

Generally, a finite family $\left(L_{i}, i \in I\right)$ of functions $L_{i}$ on a set $S$ is called a positive decomposition of unit if all these functions $L_{i}$ are non-negative and their sum $\sum_{i} L_{i}$ is equal to the function $1_{S}$ identically equal to 1 . One can understand such a family as a classical fuzzy measurement device with the set $I$ of outcomes; in a state $s$ of nature this measurement 
gives the outcome $i$ with probability $L_{i}(s)$. If we associate a prize $x_{i}$ to outcome $i$, we obtain a horse lottery $L=\sum_{i} x_{i} \otimes L_{i}$.

\section{Quantum lotteries}

A quantum lottery is also a bet on the outcome of a measurement, but now a quantum one. A measurement of some 'observable' is performed, and, depending on the result obtained, our DM receives some prize. To formalize the notion of quantum measurement we have to modify the notion of a state space. The set $S$ is replaced by some Hilbert space $H$. The notion of function on $S$ is replaced by the notion of Hermitian operator Below we give precise definitions (a reminder of elementary notions about Hilbert spaces is provided in Appendix 1). For now we only say that the main difference with the classical state space model is that the Hilbert space model allows for measurements that cannot be performed simultaneously i.e., they are incompatible with each other. Therefore the performance of a measurement can modify the state of the system.

\section{Quantum measurement}

A quantum measurement device is modeled by a finite collection $\left(P_{i}, i \in I\right)$ of Hermitian operators such that

a) all $P_{i}$ are nonnegative, and

b) $\sum_{i} P_{i}=E$.

In Physics such a collection is called POVM (positive operator valued measure); we prefer to speak about positive decomposition of unit (PDU). The elements of $I$ are the possible outcomes of the device; the operators $P_{i}$ express the potentiality for realization of the outcome $i$ in a way similar to the functions $L_{x}$ for horse lotteries (see above).

We shall distinguish between two classes of measurements. The first one consists of von Neumann measurements (they are known also as direct measurements, orthogonal measurements, first kind measurements, and reproducible measurements). They are defined by the requirement that the $P_{i}$ are orthogonal each other, that is $P_{i} P_{j}=0$ for $i \neq j$. It is easy to see that in this case all operators $P_{i}$ are projectors. Conversely, it can be shown that if all $P_{i}$ are projectors, they are orthogonal each other.

The second and broader class of measurements includes $\left(Q_{i}, i \in I\right)$ such that $Q_{i}$ commute with each other, that is $O_{i} Q_{j}=Q_{j} Q_{i}$ for any $i, j \in I$. We call such measurements internally 
consistent. It is easy to see that orthogonal operators commute, so that von Neumann measurements are internally consistent.

Examples of such measurements are 'von Neumann measurements with noise'. We make a von Neumann measurement $\left(P_{i}, i \in I\right)$ (with orthogonal $P_{i}$ ) and after having obtained an outcome $i$, we use a roulette lottery $l_{i}$ with values in a set $X$ to determine the final outcome $x$. Such a measurement device is modeled by the collection $\left(Q_{x}, x \in X\right)$, where $Q_{x}=\sum_{i} l_{i}(x) P_{i}$. Conversely, any internally consistent measurement can be represented as von Neumann measurement with noise.

An example of more general measurements is provided by the notion of a compound measurement. Suppose we have two von Neumann measurements devices, $\mathcal{P}=\left(P_{i}, i \in I\right.$ and $\mathcal{Q}=\left(Q_{j}, j \in J\right)$. Then we can form the compound measurement $\mathcal{P} \mathcal{Q}$ with the set of outcomes $I \times J$ : we perform first measurement $\mathcal{P}$, then perform $\mathcal{Q}$ and write the obtained outcomes $(i, j)$. The corresponding $\mathrm{PDU}$ is $\left(P_{i} Q_{j} P_{i}, i \in I, j \in J\right)$. If $P_{i}$ commute with $Q_{j}, \mathcal{P} \mathcal{Q}$ is von Neumann measurement as well. However in the general case, when $\mathcal{P}$ and $\mathcal{Q}$ are incompatible, the obtained measurement $\mathcal{P} \mathcal{Q}$ is not von Neumann measurement. This construction is one of the justifications for considering non-orthogonal measurements. Another line of justification relates to the possibility of defining mixtures and restrictions.

\section{Quantum Lottery}

As we already wrote, a quantum lottery is a bet on the outcome of a quantum measurement. More precisely, a Q-lottery is a pair made of a quantum measurement device $\mathcal{P}=\left(P_{i}, i \in I\right)$ (the base of the lottery) and the prizes associated with the corresponding outcomes $\left(x_{i}, i \in I\right)$. We write such a Q-lottery as $\sum_{i} x_{i} \otimes P_{i}$. Intuitively the measurement $\mathcal{P}$ is performed and depending on the outcome $i$ that obtains, the agent receives prizes $x_{i}$. The set of Q-lotteries is denoted as $\mathrm{QL}(\mathrm{H})$.

$A$ constant Q-lottery is a lottery of the form $x \otimes E$; it gives the prize $x$ with certainty.

Any Q-lottery $\sigma=\sum_{i} x_{i} \otimes P_{i}$ can be written in the canonical form $\sum_{x} x \otimes Q_{x}$, where $Q_{x}=\sum_{i, x_{i}=x} P_{i} 5$ Let $\mathbf{Q L}_{c}(H, X)$ be the set of canonical Q-lotteries (or simply $\mathbf{Q L}_{c}(H)$ because the specification of the set $X$ does not play an essential role). Intuitively, the initial Q-lottery $\sigma$ and its canonical form only differ in the way we write them and therefore can

\footnotetext{
${ }^{5}$ Although the set $X$ can be infinite only a finite number of $Q_{x}$ differ from 0 .
} 
be considered as equivalent. Below we formulate this equivalence as our 'no-framing' axiom $\mathrm{A} 0$.

As it is the case for classical lotteries, we can define mixtures of Q-lotteries, but we restrict the mixture operation to canonical lotteries. Suppose that we have two Q-lotteries in the canonical form: $\sigma=\sum_{x} x \otimes P_{x}$ and $\tau=\sum_{x} x \otimes Q_{x}$. Then we can construct a new canonical Q-lottery as the mixture of the two (with weights $\alpha$ and $1-\alpha) \alpha \sigma+(1-\alpha) \tau:=$ $\sum_{x} x \otimes\left(\alpha P_{x}+(1-\alpha) Q_{x}\right)$. The new lottery is interpreted as follows. First you use a "roulette device" to determine which one of $\sigma$ or $\tau$ will be played and thereafter you play one of them 6 (For instance, if $x^{*} \in X$ is an outcome never paid by $\tau$, then $Q_{x^{*}}=0$ and $\alpha \sigma+(1-\alpha) \tau$ pays $x^{*}$ under the event $\alpha P_{x^{*}}$.) The set $\mathbf{Q L}_{c}(H, X)$ of canonical Q-lotteries is a convex space. We shall use this structure intensively in what follows.

\section{Construction and characterization of 'nice' prefer- ences}

We are interested in preference relations over quantum lotteries that satisfy some 'natural' properties, familiar from von Neumann and Morgenstern, Savage, Aumann and others. We call such preference relations "nice". We start with a straightforward construction of nice preferences, thereafter we formulate their properties (axioms), and finally we show that these properties fully characterize nice preferences.

In order to construct preferences (in fact, to construct the utility of Q-lotteries), we should specify two things. First, a utility function $u: X \rightarrow \mathbb{R}$. Second, a linear 'belief' functional $\beta: \operatorname{Herm}(H) \rightarrow \mathbb{R}$ which is

a) positive in the sense that $\beta(A) \geq 0$ for $A \geq 0$,

b) normalized in the sense that $\beta(E)=1$.

With these two ingredients, we can define the ' $(u, \beta)$-utility' $U(\sigma)=U_{u, \beta}(\sigma)$ of any

\footnotetext{
${ }^{6}$ In the quantum case we do not in general have the equivalence - as in the classical case - with the alternative interpretation of the mixture: play both lotteries and use the roulette device to select which outcome determines the prize afterwards. This is because in the general case the two measurements implicit in the lotteries need not be compatible.
} 
Q-lottery $\sigma=\sum_{i} x_{i} \otimes P_{i}$ as

$$
U(\sigma)=\sum_{i} u\left(x_{i}\right) \beta\left(P_{i}\right)
$$

For Q-lotteries $\sigma$ and $\tau$ we set $\sigma \preceq \tau$ if $U(\sigma) \leq U(\tau)$. Below we list some 'nice' properties A0-A4 that this preference relation $\preceq$ on $\mathrm{QL}(H)$ possesses.

No framing

A0. For any $\sigma, \tau \in \mathrm{QL}(H)$ with respective canonical forms $\sigma^{\prime}$ and $\tau^{\prime}, \sigma \preceq \tau \Leftrightarrow \sigma^{\prime} \preceq \tau^{\prime}$.

Axiom A0 follows from the fact that the utility of a Q-lottery $\sigma=\sum_{i} x_{i} \otimes P_{i}$ is equal to the utility of its canonical form $\sum_{x} x \otimes\left(\sum_{i, x_{i}=x} P_{i}\right)$. Indeed, the utility of the latter is equal to $\sum_{x} u(x) \beta\left(\sum_{i, x_{i}=x} P_{i}\right)=\sum_{x} \sum_{i, x_{i}=x} u\left(x_{i}\right) \beta\left(P_{i}\right)=\sum_{i} u\left(x_{i}\right) \beta\left(P_{i}\right)=U(\sigma)$.

This axiom is implicit in the Savage and Anscombe-Aumann frameworks. However, in generalizations of these frameworks it must be imposed explicitly see e.g., Cohen and Jaffray (1980). They formulate an axiom of 'non influence of formalization' very similar to our axiom A0. There are also other works that reject that axiom in order to allow for framing effects see Ahn and Ergin (2010).

\section{Weak order}

A1. The preference relation $\preceq$ is a weak order, that is complete and transitive.

This follows from its representation via the utility $U$.

The next following two properties assert that the preference relation is consistent with a mixture structure on the set $\mathbf{Q L}(H)$.

\section{Independence}

A2. Let $\sigma, \tau, \varphi \in \mathbf{Q L}_{c}(H)$ be Q-lotteries, and $\alpha \in[0,1]$. If $\sigma \preceq \tau$ then $\alpha \sigma+(1-\alpha) \varphi \preceq$ $\alpha \tau+(1-\alpha) \varphi$.

Property A2 follows from the linearity of $\beta$.

\section{Continuity}

A3. Let $\sigma, \tau, \varphi \in \mathbf{Q L}_{c}(H)$, and $\sigma \prec \varphi \prec \tau$. Then there exists $\alpha$ and $\beta(0<\alpha, \beta<1)$ such that $\alpha \sigma+(1-\alpha) \tau \prec \varphi$ and $\varphi \prec \beta \sigma+(1-\beta) \tau$. 
Indeed, since $U(\sigma)<U(\varphi)<U(\tau)$, we have $U(\varphi)>\alpha U(\sigma)+(1-\alpha) U(\tau)$ for some $\alpha \in(0,1)$ and $U(\varphi)<\beta U(\sigma)+(1-\beta) U(\tau)$ for some $\beta \in(0,1)$. So $U(\varphi)>U(\alpha \sigma+(1-\alpha) \tau)$ and $U(\varphi)<U(\beta \sigma+(1-\beta) \tau)$.

\section{Monotonicity}

This property is the most subtle. It asserts, roughly speaking, that if we in a lottery $\sigma=\sum_{i} x_{i} \otimes P_{i}$ replace the prizes $x_{i}$ with better ones then new lottery will be preferred to the initial one. However, this formulation is too weak for our aims, and we formulate it in stronger form. To do that we first note that we can define the canonical form not only for 'prize valued Q-lotteries' but also for 'roulette valued Q-lotteries', that is for expression of the form $\sum_{i} l_{i} \otimes P_{i}$, where $l_{i}=\sum_{x} x \otimes l_{i}(x)$ are roulette lotteries. The canonical form of this lottery is $\sum_{x} x \otimes Q_{x}$, where $Q_{x}=\sum_{i} l_{i}(x) P_{i}$. Let $\mathbf{Q L}(H, \Delta(X))$ denote the set of all roulette-valued Q-lotteries. We use this to extend the preference relation to $\mathrm{QL}(H, \Delta(X))$ as follows: for any $\sigma, \tau \in \mathrm{QL}(H, \Delta(X))$ with respective canonical form $\sigma^{\prime}$ and $\tau^{\prime}, \sigma \preceq \tau$ if and only if $\sigma^{\prime} \preceq \tau^{\prime}$. We next have to consider the derived preference relation $\preceq_{\Delta}$ on the set of ordinary (roulette) lotteries $\Delta(X)$. For roulette lotteries $l$ and $m$ we set $l \preceq_{\Delta} m$ if $l \otimes E \preceq m \otimes E$. Here $l \otimes E$ denotes a constant Q-lottery, getting with certainty the prize $l$, and similarly for $m \otimes E$.

Note that the preference $\preceq_{\Delta}$ on $\Delta(X)$ is represented by the affine extension of $u$ from $X$ to $\Delta(X)$, that we still denote by $u$. Indeed, we have $U(l \otimes E)=U\left(\sum_{x} x \otimes l(x) E\right)=$ $\sum_{x} u(x) \beta(l(x) E)=\sum_{x} u(x) l(x)=u(l)$, because $\beta(E)=1$.

A4. Let $\sigma=\sum_{i} l_{i} \otimes P_{i}$ and $\tau=\sum_{i} m_{i} \otimes P_{i}$ be elements of $\mathrm{QL}(H, \Delta(X))$ with the same base. If $l_{i} \preceq_{\Delta} m_{i}$ for any $i \in I$ then $\sigma \preceq \tau$.

This property can be considered as a strong version of the sure-thing principle of Savage. It is a simple consequence of the formula $U(\sigma)=\sum_{i} u\left(l_{i}\right) \beta\left(P_{i}\right)$ and of the non-negativity of $\beta\left(P_{i}\right)$.

Definition 3. A preference relation $\preceq$ on the set $\mathrm{QL}(H)$ of Q-lotteries is nice if it has the properties A0 - A4 (or satisfies the axioms A0 - A4).

The discussion above shows that a preference relation $\preceq_{u, \beta}$ built with the help of a utility function $u$ on $X$ and a linear functional $\beta$ on $\operatorname{Herm}(H)$ is nice. Our first result asserts that 
the reverse is also true.

Theorem 1. A preference relation $\preceq$ on $\mathrm{QL}(H)$ is nice if and only if there exist a function $u$ on $X$ and a positive and normalized linear functional $\beta$ on $\mathbb{P}(H)$ such that $\preceq=\preceq u, \mu$.

Moreover, if the preference $\preceq$ is not trivial (that is there exist Q-lotteries $\sigma$ and $\tau$ such that $\sigma \prec \tau)$ then $\beta$ is unique and $u$ is unique up to a positive affine transformation.

The proof is in Appendix 2. We now briefly sketch this proof. First, fix a measurement device $\mathcal{P}=\left(P_{i}, i \in I\right)$. In the first stage of the proof, we use the primitive preference $\preceq$ on $\mathbf{Q L}(H)$ to construct another preference $\preceq_{\mathcal{P}}$ on the set $\mathbf{Q L}_{\mathcal{P}}(H, \Delta(X))$ of roulette-valued Q-lotteries with base $\mathcal{P}$. Viewing each such Q-lottery in $\mathbf{Q L}_{\mathcal{P}}(H, \Delta(X))$ as an AnscombeAumann act from $I$ to $\Delta(X)$, we invoke the Anscombe-Aumann (1963) theorem, and obtain a utility function $u_{\mathcal{P}}$ and a probability vector $\beta_{\mathcal{P}}$ on $I$ providing together a Subjective Expected Utility (SEU) representation of $\preceq_{\mathcal{P}}$. In the second stage of the proof, we show that these various SEU represetations are consistent with each other; that is, that the functions $u_{\mathcal{P}}$ are essentially independent of $\mathcal{P}$ and that the probability vectors $\beta_{\mathcal{P}}$ arise from a single belief functional $\beta$.

In a classical Anscombe-Aumann setup, it is straightforward to obtain the consistency of the various SEU representations accross different partitions of the state space because acts are directly defined as functions from the state space to the the roulette space. But suppose now that an act is rather a structure $\left(E_{1}, l_{1} ; \ldots ; E_{n}, l_{n}\right)$ where $\left(E_{1}, \ldots, E_{n}\right)$ is a partition of the state space and $\left(l_{1}, \ldots, l_{n}\right)$ is a corresponding collection of lotteries on $X$. Such an act induces a function $\sum_{i} \mathbb{1}_{E_{i}} l_{i}$. Under an additional axiom requiring two acts inducing the same function to be always indifferent, again we can easily obtain the consistency of the various SEU representations accross the various partitions of the state space. Comming back at our nonclassical setup, it takes a very similar axiom, namely A0, to obtain the consistency of the SEU representations $\left(u_{\mathcal{P}}, \beta_{\mathcal{P}}\right)$, accross the various PDU. And from there the full representation stated in Theorem 1 easily follows. We next investigate some consequences of our result and some reformulations.

The shadow operator of a Q-lottery

Let $\preceq$ be a nice preference relation on $Q$-lotteries. We fix some function $u$ from $X$ 
to $\mathbb{R}$. We call the shadow operator of a lottery $\sigma=\sum_{i} x_{i} \otimes P_{i}$ the Hermitian operator $S h(\sigma)=S h^{u}(\sigma)$ defined as follows

$$
S h(\sigma)=\sum_{i} u\left(x_{i}\right) P_{i}
$$

We note that $\beta(S h(\sigma))=U(\sigma)$, and consequently the utility of Q-lottery $\sigma$ depends only on its shadow operator. The notion of shadow operator is the equivalent of the notion of utility profile in the classical framework. And in the same way the expected utility of an act only depends on its utility profile. From here we could completely forget about Q-lotteries and discuss the utility of Hermitian operators expressed by the functional $\beta$.

\section{Trace and belief operator}

There exists a remarkably useful way of representing the belief functional $\beta$ by means of a (Hermitian) operator of belief. For that we shall make extensive use of the concept of the trace of an operator (and precisely here the finite dimensionality of $H$ becomes important). A reminder of the definition and properties of the trace is provided in Appendix 1. Of particular value in our context are two properties: commutativity $\operatorname{Tr}(A B)=\operatorname{Tr}(B A)$ and the fact that the trace of any Hermitian operator is a real number.

Definition 4. We call a belief operator (or a cognitive state) any nonnegative Hermitian operator with the trace equal to 1.

Given a belief operator $B$, we can define the functional $\beta$ on $\operatorname{Herm}(H)$, setting $\beta(A)=$ $\operatorname{Tr}(A B)$ for any $A \in \operatorname{Herm}(H)$.

Lemma 1. 1) The functional $\beta$ takes real values;

2) $\beta(A) \geq 0$ for $A \geq 0$;

3) $\beta(E)=1$.

Proof. See Lemma in Appendix 1.

As a consequence, we obtain that the functional $\beta$ built on the belief operator $B$ is a belief functional. Moreover any belief functional has such a form (for a unique belief operator $B$ ). In fact the formula $(A, B)=\operatorname{Tr}(A B)$ gives a scalar product (and thereby also the structure of an Euclidean space) on the real vector space Herm $(H)$. Relying on well-known description of linear functionals on Euclidean space, we have proved the following 
Theorem 1'. A preference relation $\preceq$ on $\mathrm{QL}(H)$ is nice if and only if there exist a utility function $u: X \rightarrow \mathbb{R}$ and a belief operator $B$ such that $\preceq$ is represented by the function $\sigma \mapsto \operatorname{Tr}\left(S h^{u}(\sigma) B\right)$

Moreover, if the preference $\preceq$ is not trivial (that is there exist Q-lotteries $\sigma$ and $\tau$ such that $\sigma \prec \tau$ ) then $B$ is unique and $u$ is unique up to a positive affine transformation.

Remark. In quantum physics such belief operators are called 'states' or 'density operators'. We shall refer to them as simply 'beliefs' or 'cognitive state' because they allow constructing subjective probabilities in a most suitable way. Indeed, let $B$ be a belief operator; then, for any event $P, \beta_{B}(P)=\operatorname{Tr}(P B)$ is the subjective probability for event $P$ when the cognitive state is $B$.

Example 1. Assume that belief operator $B$ is given as the projector $P=P_{e}$ on a one-dimensional subspace $\mathbb{C} e \subset H$, generated by a vector $e$ of length 1 (that is $(e, e)=1$; in Physics such operators are called pure states). In other words, $P(x)=(x, e) e$. It is easy to check that, for any Hermitian operator $A$ (viewed as a Q-lottery), its utility $U(A)$ is equal to $\operatorname{Tr}(A P)=(A e, e)$. In other words, the quadratic form $(A e, e)$ gives the utility of lottery $A$ when the beliefs are represented by a pure state $e$.

Such beliefs correspond to a maximally precise (subjective) representation of the state of measured system 7

Example 2. Let us now consider the opposite case, when DM has beliefs represented by the operator $B=E / \operatorname{dim} H$. Such beliefs can be interpreted as 'uniform uncertainty'; roughly speaking, the DM assigns equal probability to all states. The expected value of a lottery $A$ under such beliefs is $U(A)=\operatorname{Tr}(A E) / \operatorname{dim}(H)=\operatorname{Tr}(A) / \operatorname{dim}(H)$ which corresponds to the arithmetic average of the eigenvalues of operator $A$.

Note that whatever the beliefs $B$, the expected value of a lottery-operator $A$ equals to some convex combination of its eigenvalues.

\footnotetext{
${ }^{7}$ In the quantum context, a (pure) maximal information information state is not a complete information as in the classical context. In a maximal information state any measurement that generates new information leads to the loss of some other previously known information: the state changes.
} 


\section{Updating}

Suppose that beliefs of a DM are given by a belief operator $B$. That is, our decision-maker believes that the state of the measured quantum system is $B$. Alternatively, her cognitive state is such that she assigns probabilities to events according to $B$. This means the following. We consider here events as subspaces of $H$, or as projectors. If $P$ is a projector then the probability of $P$ (at the belief $B$ ) is equal to $\operatorname{prob}(P)=\operatorname{Tr}(P B)$. Since $P$ is a projector, we can rewritten the latter as $\operatorname{Tr}(P B P)$. In the next section we return to the interpretation of $B$. But for now suppose that she receives information of relevance for the lotteries. This information may concern the prizes or be relative to the system, the measurements of which determines the outcomes and the prizes. In the following we restrict ourselves to the case when the value of the prizes does not change (and is given by a fixed utility function $u$ ) and all new information concerns the measured system. For instance, the DM (or someone else) performs some intermediate measurement and learns as a result of the measurement that some event $P$ occurred. It is almost obvious that her beliefs and preferences on Q-lotteries should change, the question we ask in this section is how should her preferences on quantum lotteries change after receiving that information?

In Quantum Mechanics, it is simply postulated that states change in accordance with the von Neumann-Lüders postulate. More precisely, a system that was in state $B$ transits to the state $B^{\prime}=P B P / \operatorname{Tr}(P B P)$ as a result of performing a measurement that yields the event $P$. The operator $P B P$ is Hermitian and non-negative $((P B P v, v)=(B P v, P v) \geq 0$ by force of the nonnegativity of $B$ ). Thus, $B^{\prime}$ is indeed a state. Here, we need to clarify why $\operatorname{Tr}(P B P)$ is different from zero so we are allowed to divide by this number. As a matter of fact, we understand $\operatorname{Tr}(P B P)$ as the probability to discover event $P$ in cognitive state $B$. Thus, by analogy with standard Bayesian updating, the von Neumann-Lüders postulate focuses on cases where the state assigns a positive probability to event $P$. If the trace $\operatorname{Tr}(P B P)$ were equal to 0 , that would mean that something happened which has zero probability, i.e. an event that is considered impossible under belief $B$. That is, the beliefs of our DM captured by the state $B$ are simply incorrect and she has to update them in a more fundamental way.

We want to show that in quantum decision theory beliefs change in the same way. Clearly, we have to make some assumptions. In order to determine which assumptions we need, we 
return for a minute to the behavior of a classical decision-maker. She has preference between functions (acts) defined on the set $S$ of states of nature; suppose that she learns in addition that the true state lies in some subset $T \subseteq S$. It is quite natural to assume that her new preference depends only on values of these functions on the subset $T$. That is, only on the restriction of the various functions to $T$.

\section{Information as events}

We want to proceed with Q-lotteries in a way analogous to the classical case. We shall assume that the new information comes from the performance of a projective measurement and that the obtained result informs that event $P$ has occurred. Here $P$ is a projector on subspace $W$. We have to define what we mean with the "restriction of a Q-lottery" on a subspace $W$.

As a subspace of the Hilbert space $H, W$ is also a Hilbert space. Given an Hermitian operator $A$ on $H$, one can consider the operator $P A$ as an operator on $W(v \mapsto P A(v)$ for $v \in W$ ). To avoid a confusion, we denote operator $P A$, conceived as an operator on $W$, by $A \mid W$ and call it the restriction of $A$ on $W$. First we note that $A \mid W$ is an Hermitian operator (as an operator on $W$, not on $H$ ). Indeed, if $y, z \in W$ (and hence $P y=y, P z=z$ ) then

$$
(P A y, z)=(A y, P z)=(A y, z)=(y, A z)=(P y, A z)=(y, P A z)
$$

The same argument shows that $A \mid W$ is a nonnegative operator on $W$ provided $A$ is nonnegative. Note, finally, that $P \mid W$ is the identity operator on $W$.

The above indicates how to define a restriction to subspace $W$ of any Q-lottery. If $\sigma=\sum_{i} x_{i} \otimes P_{i}$ is a Q-lottery on $H$, then $\sigma\left|W:=\sum_{i} x_{i} \otimes P_{i}\right| W$ is a Q-lottery on $W$. We call $\sigma \mid W$ the restriction of lottery $\sigma$ to $W$. Clearly, $S h(\sigma \mid W)=S h(\sigma) \mid W$.

Let us next turn to the problem of updating nice preferences. Suppose that $\preceq$ is a nice preference relation on the set $\mathbf{Q L}(H)$; due to Theorem $1^{\prime}$ it is given by some belief operator $B$. Suppose now that our DM receives information in the form of an event-subspace $W \subset H$ (or of an event-projector $P$ ). The updated preference relation will be denoted as $\preceq_{W}$. Following Ghirardato (2002), we formulate two axioms connecting $\preceq_{W}$ and $\preceq$. The first one A5 is 'consequentialism': 
A5 If $\sigma$ and $\tau$ are Q-lotteries on $H$, and $\sigma|W=\tau| W$, then $\sigma$ and $\tau$ are equivalent with respect to $\preceq_{W}$.

To formulate the second axiom we need one more notion. We say that a projector $P$ is compatible with a Q-lottery $\sigma=\sum_{i} x_{i} \otimes P_{i}$ if $P$ commutes with every $P_{i}$. Now we can formulate axiom $\mathbf{A} \mathbf{6}$ of compatible dynamic consistency:

A6 Suppose that lotteries $\sigma$ and $\tau$ are compatible with $P$, and $\sigma\left|W^{\perp}=\tau\right| W^{\perp}$, then $\sigma \preceq_{W} \tau$ if and only if $\sigma \preceq \tau$.

Extending Ghirardato (2002) to the quantum context, we state the following

Theorem 2. Let $\preceq$ be a non-trivial nice preference given by a belief operator $B$, and $\operatorname{Tr}(P B P)>0$. Then

a) The preference relation $\preceq_{W}$ given by the belief operator $B^{u p}=P B P / \operatorname{Tr}(P B P)$ satisfies axioms $\boldsymbol{A} \mathbf{5}$ and $\boldsymbol{A} \boldsymbol{6}$.

b) Conversely, if a preference relation $\preceq_{W}$ satisfies Axioms $\boldsymbol{A 1}, \boldsymbol{A 5}$ and $\boldsymbol{A 6}$, then it is nice and it is given by the 'updated' belief operator $B^{u p}=P B P / \operatorname{Tr}(P B P)$.

The proof of Theorem 2 is in Appendix 3. It generalizes the classical equivalence result between Dynamic Consistency and Consequentialism on the one hand and Bayesian updating on the other hand to our nonclassical setup. Its proof is similar to the classical one. Given two classical Q-lotteries $\sigma, \tau \in \mathbf{Q L}(H)$, we construct two other Q-lotteries $a d_{W}(\sigma), a d_{W}(\tau) \in$ $\mathrm{QL}_{c}(H)$ with the following properties:

(1) $a d_{W}(\sigma)|W=\sigma| W$ and $a d_{W}(\tau)|W=\tau| W$,

(2) $a d_{W}(\sigma)\left|W^{\perp}=a d_{W}(\tau)\right| W^{\perp}$.

That is, $a d_{W}(\sigma)$ and $a d_{W}(\tau)$ agree on $W$ with $\sigma$ and $\tau$ respectively, while they agree with each other on $W^{\perp}$. Given Axioms A5 and A6, these two properties imply the equivalence between $\sigma \preceq_{W} \tau$ and $a d_{W}(\sigma) \preceq a d_{W}(\tau)$ for any Q-lotteries $\sigma$ and $\tau$. In a classical setup, this equivalence is essentially a form of Savage's (1954) Sure Thing Principle. Actually, Savage postulates a preference satisfying the Sure Thing Principle and defines conditional preference through this equivalence. Moreover, we show that we can choose $a d_{W}(\sigma)$ and $a d_{W}(\tau)$ such that their shadow operators are respectively given by $P S h(\sigma) P$ and $P S h(\tau) P$. In classical terms, this means that the 'utility profiles' induced by $a d_{W}(\sigma)$ and $a d_{W}(\tau)$ are equal to those of $\sigma$ and $\tau$ on event $W$ and equal to 0 otherwise. From there, it takes a little algebra 
and the uniqueness part of Theorem 1' to conclude. We next discuss some corollaries of this theorem.

Remark 1. We above assumed that the probability of the event $P$ is non-zero. In the opposite case the received information contradicts the initial beliefs. Consider now the case when the probability of the event $P$ (equal, as we know, to $\operatorname{Tr}(P B P)$ ) is 1 , that is our DM is sure that event $P$ must occur. Receiving information that $P$ occurred she does not learn anything and intuitively we expect her preferences to remain the same, which indeed obtains:

Proposition 1. A distance between a prior $B$ and the posterior $B^{u p}$ is $O\left((1-\operatorname{Tr}(P B P))^{1 / 4}\right)$.

In particular, if $\operatorname{Tr}(P B P)=1$ then $B^{u p}=B$. We prove Proposition 1 in Appendix 4 .

Remark 2. Let us consider a situation when the initial (a priori) beliefs are maximally precise, that is, they are given (as in Example 1) by a one-dimensional projector or, to put it differently, $B$ is a pure state. How does the DM update her beliefs as she receives new information? We expect the updated state to be pure as well. This is true in the classical context and this is true in the quantum case as well with a noticeable distinction that the new pure state is generally not the same as the initial one.

Proposition 2. If a belief operator $B$ is a one-dimensional projector then the updated operator $B^{u p}$ is a one-dimensional projector as well.

Proof. The rank of operator $P B P$ is less or equal to the rank of operator $B$, that is $\leq 1$.

The main difference with the classical situation is that the new state is generally different from the initial one. Indeed, let our belief operator $B$ be the projection on the line $\mathbb{C} e$, where $e$ is a normalized vector $((e, e)=1)$. Such a projector $B$ moves an arbitrary vector $x$ to vector $(x, e) e$. Then the operator $P B P$ moves vector $x$ to $P((P x, e) e)=(P x, e) P(e)=(x, P e) P e$, that is the projection (up to the multiplier $(P e, P e)$ ) on the line $\mathbb{C} P e$. Thus the pure state $e$ is changed into another pure state $P e / \sqrt{(P e, P e)}$. The reader can see here the projection postulate at work. We would like to add that the modification is smaller the closer the vector e to the subspace $W$ associated with the projector $P$. That is the closer $\operatorname{Tr}(P B P)=(P e, P e)$ to 1. 
Remark 3. Suppose that, after receiving information in the form of an event-projector $P$, the DM receives yet some new piece of information in the form of an event-projector $Q$ (which does not contradict $P$ ). Then (up to a factor) the state changes as $B \mapsto P B P \mapsto Q P B P Q$. However, in the case when events-projectors do not commute with each other, the updated beliefs depend on the order in which the updating is realized. Manipulating the order in which information is provided affects our DM preferences and thus her choice behavior. As we shall see later, together with Remark 2, Remark 3 paves the ground for interesting economic implications.

Remark 4. Above we assume that the information comes in the form of an event, that is a subspace $W$ or a projector $P$. However we could consider the more general case when the information has the form a 'fuzzy-projector', that is an Hermitian operator $P$ such that $0 \leq P \leq E$. In this case the updating of the belief take the form $B^{u p}=\sqrt{P} B \sqrt{P} / \operatorname{Tr}(P B)$. Proposition 1 generalizes to this setting; see Appendix 4.

\section{Information as measurements}

In the remaining of the paper we use the 'shadow operator' expression for lotteries: $A=S h^{u}(\sigma)$. Due to Theorem $1^{\prime}$, the utility of such a lottery under belief $B$ is equal to $U(A)=\operatorname{Tr}(A B)$.

Usually, new information arises as an outcome of some measurement. Suppose we perform some von Neumann measurement, represented by an orthogonal decomposition of unit, ODU $\left(P_{i}, i \in I\right)$. If, as the result of this measurement, we obtain outcome $i$, the belief-state $B$ changes (is updated) into $B_{i}=P_{i} D P_{i} / \operatorname{Tr}\left(P_{i} B\right)$. We say that the state of the system transits into subspace $W=\operatorname{Im}\left(P_{i}\right)$. Note that the number $p_{i}=\operatorname{Tr}\left(P_{i} B\right)$ is precisely the probability (under the belief $B$ ) for the realization of outcome $i$ when performing our measurement. Excluding impossible results, we can assume that these numbers are stricty positive.

The utility of 'lottery' $A$, after the DM received information about realization of outcome $i$, is now equal to $U_{i}(A)=\operatorname{Tr}\left(A B_{i}\right)$. It may clearly be either larger or smaller than the initial $U(A)$.

Further we discuss an interesting case when such an 'informational' measurement has been performed (using ODU $\mathcal{P}=\left(P_{i}, i \in I\right)$ ), but its outcome is not known to the DM, i.e., she only knows that the measurement took place. This is a new type of information; 
in Physics one speaks about 'decoherence'. It is a fully non-classical phenomenon, because in the classical situation such an 'information' is useless. This is not the case in a quantum context.

We earlier established that when learning about the occurrence of an outcome $i$, the utility of the lottery-operator $A$ is updated to $U_{i}(A)=\operatorname{Tr}\left(A B_{i}\right)$ (where $B$ is the belief of our DM, and $\left.B_{i}=P_{i} B P_{i} / \operatorname{Tr}\left(P_{i} B\right)\right)$ that is equal to $\operatorname{Tr}\left(A P_{i} B P_{i}\right) / p_{i}$. Since the probability of outcome $i$ is $p_{i}$, then the expected utility of our lottery $U^{\prime}(A)$ (when learning about the performance of measurement $\mathcal{P}$ ) is

$$
U^{\prime}(A)=\sum_{i} p_{i} U_{i}(A)=\sum_{i} \operatorname{Tr}\left(A P_{i} B P_{i}\right)
$$

(Of course, this means also that the state of belief $B$ has changed into a new state $B^{\prime}=$ $\left.\sum_{i} P_{i} B P_{i}\right)$. And, as we shall see, the connection between these two utilities, i.e. ex-ante $U(A)$ and ex-post $U^{\prime}(A)$, is not straightforward in general. Ex-post utility can be either larger or smaller than the initial (a priori) utility as the following simple example shows.

Example 3. Let $H$ be a two dimensional Hilbert space with orthonormal basis $\left(e_{1}, e_{2}\right)$. Let $A$ be a projector on $e_{1}$, i.e., an operator of the form $A=\left(\begin{array}{ll}1 & 0 \\ 0 & 0\end{array}\right)$. Consider $A$ as a lottery that gives a utility equal to 1 in state $e_{1}$ and 0 otherwise. Consider another lottery-operator $C=\left(\begin{array}{ll}0 & 0 \\ 0 & 1\end{array}\right)$ that gives utility 1 in state $e_{2}$ and 0 otherwise. Assume now that our DM's belief-state is given by $B=A$. Then clearly the expected utility of lottery $A$ is equal to 1 and the expected utility of $C$ is equal to zero. So our DM strictly prefers $A$ to $C$.

Assume now that we perform a measurement defined by the ODU $\left(P_{1}, P_{2}\right)$, where $P_{1}=$ $\left(\begin{array}{ll}1 / 2 & 1 / 2 \\ 1 / 2 & 1 / 2\end{array}\right)$ and $P_{2}=\left(\begin{array}{cc}1 / 2 & -1 / 2 \\ -1 / 2 & 1 / 2\end{array}\right)$. If the outcome of the measurement is 1 , the updated belief-state is given by operator $B_{1}=P_{1} B P_{1} / \operatorname{Tr}\left(P_{1} B P_{1}\right)$, which as can be seen easily is equal to $P_{1}$. The expected utility in the belief-state is $U_{1}(A)=\operatorname{Tr}\left(A B_{1}\right)=\operatorname{Tr}\left(A P_{1}\right)=$ $1 / 2$. And similarly if we obtain the complementary result 2 , the belief-state is updated to $B_{2}=P_{2} B P_{2} / \operatorname{Tr}\left(P_{2} B P_{2}\right)$ and the corresponding expected utility is $U_{2}(A)=\operatorname{Tr}\left(A B_{2}\right)=$ $\operatorname{Tr}\left(A P_{2}\right)=1 / 2$. So we see that for any one of the two possible outcomes the value of the $A$ lottery goes from 1 to $1 / 2$. With the same reasoning we obtain that $U_{1}(B)=\operatorname{Tr}\left(C B_{1}\right)=$ $\operatorname{Tr}\left(C P_{1}\right)=1 / 2=U_{1}(C)$. So the two lotteries $A$ and $C$ yield the same expected utility. This 
violates "recursive dynamic consistency": lottery $A$ is ex-post indifferent to $C$ whatever the outcome of the measurement, yet ex-ante it is strictly preferred. Note that the intermediary measurement $\left(P_{1}, P_{2}\right)$ is incompatible with either $A$ or $C$ and $B$.

It may seem odd that our dynamic consistency axiom A6 allows for such departures. But the appeal of recursive dynamic consistency is based upon the implicit assumption that "the act the agent performs has no effect on the resolution of uncertainty" (cf. Fishburn, 1970). However, the resolution of uncertainty is - in our setting - affected by the act that is selected and the measurements it entails (as well as by other measurements) performed to acquire new information. Once this is taken into account, recursive dynamic consistency loses much of its appeal. In the next section we discuss an economic example of the phenomenon exhibited above and establish its connection with the behavior documented in the "Economics of Manipulation and Deception" (Akerlof and Schiller, 2015).

Example 3 allows illustrating 'information as measurements' a feature that lacks counterpart in the classical model. Imagine that we perform the measurement described above but our DM is not informed of the result. She only knows the measurement has been made. In the classical world such an information does not affect the DM's belief or the expected value of the lotteries. However in the quantum context the DM understands that for any of the two outcome ( 1 or 2 ) the expected value of lottery $A$ has changed from 1 to $1 / 2$. Therefore, independently of her (lack of) knowledge about the outcome of the measurement, she will revise her belief-state only because she knows that this specific measurement has been performed. The new belief-state is $B^{\prime}=p_{1} B_{1}+p_{2} B_{2}=E / 2$ which corresponds to 'uniform ignorance'. And in this belief-state the expected utility of lottery $A$ is equal to $\operatorname{Tr}\left(A B^{\prime}\right)=\operatorname{Tr}(A E) / 2=1 / 2$.

There are two interesting cases when decoherence (that is a measurement with unknown outcome) does not change the utility of a lottery or the state of belief. These are cases when the measurement $\mathcal{P}$ is compatible either with the lottery $A$ or with the belief $B$, i.e. when all the projectors $P_{i}, i \in I$, commute either with operator $A$ or with operator $B$.

Proposition 3. Assume that the intermediate measurement is compatible with either operator $A$ or $B$. Then $U(A)=U^{\prime}(A)$.

Proof. $U^{\prime}(A)=\sum_{i} \operatorname{Tr}\left(A P_{i} B P_{i}\right)=\sum_{i} \operatorname{Tr}\left(P_{i} A P_{i} B\right)$. Suppose that $P_{i} A=A P_{i}$. Then the 
second sum can be rewritten as

$$
\sum \operatorname{Tr}\left(P_{i} P_{i} A B\right)=\sum \operatorname{Tr}\left(P_{i} A B\right)=\operatorname{Tr}\left(\left(\sum P_{i}\right) A B\right)=\operatorname{Tr}(A B)=U(A)
$$

Suppose now that $P_{i} B=B P_{i}$. Then the first sum can be rewritten as

$$
\sum \operatorname{Tr}\left(A P_{i} P_{i} B\right)=\sum \operatorname{Tr}\left(A P_{i} B\right)=\operatorname{Tr}\left(A\left(\sum P_{i}\right) B\right)=\operatorname{Tr}(A B)=U(A)
$$

Remark 5. Decoherence always changes state of belief toward a more dispersed one. One can give to this statement an exact sense, using notions from the book of Alberti and Uhlmann "Stochasticity and partial order", 1982. Here we would like to illustrate this by an example when initial state is pure, presented by a (normalized) vector $e$. As we see from the formula above (for updating the belief operator), the updated state is a probability mixture (with weights $p_{i}$ ) of pure states corresponding to the projections of $e$ on the subspaces $W_{i}$. That is a pure (coherent) state disintegrates (decoheres) into a mixture of pure states.

\section{Quantum Cognition in Economics}

In this section we discuss the possible value of our results for behavioral economics. We start with a few words about quantum cognition. We know that in order to assess the world we build a representation of it, a "represented world" which is a mental construct. In classical standard theory, the represented world reflects our incomplete knowledge about the world expressed in our beliefs and these beliefs (should) evolve according to Bayes' rule in response to new information. Quantum cognition has been developed under the last decades as alternative approach to incorporate two observations: 1. People have difficulties to build a representations of a complex object. What people do is to consider a complex object from different perspectives - one at a time; 2. People may be unable to combine perspectives i.e., to synthesize all relevant information into one stable representation of the complex object. Quantum cognition models the incapacity to combine some pieces of information by analogy with incompatible properties (also called "Bohr complementary") in Quantum Mechanics: different properties may be incompatible in the sense that they cannot be given 
a determinate value simultaneously (cf speed and position) but they complement each other in the description of the system. Similarly different perspectives on an alternative may be incompatible in the mind in the sense that the individual cannot have a clear stand with respect to them simultaneously (i.e., combine them in a single coherent stable picture) but the different perspectives contribute to characterizing the alternative.

In the present paper, we have extended decision theory to a non-classical uncertainty environment. An interpretation of this move corresponds to proposing that the "represented world" used to evaluate lotteries exhibits quantum-like properties. In other words quantum indeterminacy of beliefs captures the above mentioned cognitive limitations. Our results with respect to dynamic consistency shed new light on observed behavioral anomalies in the spirit of Shiller (2000) and Akerlof and Shiller (2015) who write: "In our thoughts, as in our conversation, our minds may change. It is not just that we acquire new "information"; we change our point of view and we interpret information in a new way. Importantly these evolutions of our thoughts mean that our opinions, and the decisions that are based on them, may be quite inconsistent" (p.45). We next provide an example showing how quantum indeterminacy of beliefs and in particular its dynamic properties illustrated in Example 3 above delivers the kind of manipulability at the core of Phishing for Phools.

Consider a seller who wants to sell a new smartphone at price $300 \$$ and a customer considering buying one. Initially, the customer holds beliefs about the quality of the smartphone that can be either Excellent (utility 600) or Standard (utility 100). Her initial cognitive state assigns subjective probabilities 0.25 and 0.75 respectively to the two possible events so the expected utility is $E U(S)=.25 \cdot 600+.75 \cdot 100=225$. The alternative is to keep the money which has utility 300. So initially the customer does not want to buy the new smartphone since $225<300$. Now the seller engages in a conversation about the use of the new smartphone among famous people. In so doing the customer is moved from a private user perspective on the smartphone to a "glamour" perspective (also with two outcomes: glamour, not glamour). Assume that the two perspectives are incompatible in the mind. Now what counts to our consumer is her idol Beyoncé, so she asks whether Beyoncé uses that smartphone. The seller answers truthfully either yes or no. After the conversation the consumer updates her beliefs, her cognitive state is modified 8 Consider for simplicity the

\footnotetext{
${ }^{8}$ One possible interpretation is when in the "glamour" perspective, she is (possibly uncon-
} 
case when private use and glamour perspectives are totally uncorrelated (the corresponding bases are $45^{\circ}$ rotations of each other - see the figure below). As she turns back to the question whether or not to buy the smartphone, the lottery with updated beliefs yields $E U(S)=.5 \cdot 600+.5 \cdot 100=400>300$ whether she learned that the smartphone is glamour or not (and her cognitive state is projected onto the corresponding axis):" the phool has been phished" i.e., the seller exploited the quantum indeterminacy of the consumer's "represented smartphone" (that is her beliefs and her consistent updating rule when evaluating the lottery) to change her preferences so she chooses to purchase the smartphone. Quoting Akerlof and Shiller again "Just change people focus and you can change the decisions they make" (p. 173).

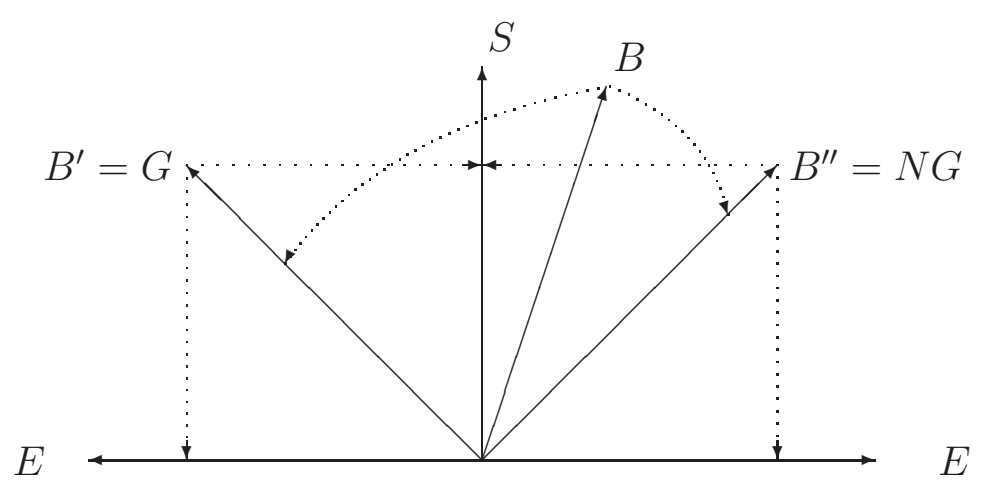

The idea of the Phishing equilibrium is that opportunities for manipulations will be exploited whenever that is profitable. By recasting the standard approach into non-classical uncertainty our theory can provide a rigorous setting for investigating the properties of competitive markets with manipulable consumers.

\section{Concluding remarks}

In this paper we provided a fully consistent choice theory integrating cognitive limitations affecting our capacity to build representations of choice alternatives. We found that the mathematical formalism of quantum mechanics offers a suitable framework for modeling such cognitive limitations. We fully characterize the rules for consistent choice behavior in a non-classical uncertainty environment. A concept of quantum lottery is introduced and

sciuosly) reminded of the general disconnect between what is temporarily "fashionable" and fundamental user values of commodities. 
sufficient and necessary conditions for choice behavior to be representable by an expected utility function are formulated in a most general setting. We also derive, from behavioral principles, an updating rule that secures the dynamic consistency of the preference relation as the decision-maker learns new information.

We found that most of the classical axioms of decision theory carry over to the context of quantum lotteries. This is because all but one axiom can be formulated in terms of a single orthogonal decomposition of the state space. When considering a single orthogonal decomposition, quantum lotteries operating in the Hilbert space are equivalent to roulette lotteries in a classical state space. An additional axiom is required to secure that the probability for any specific event does not depend on the particular lottery that it belongs to. The necessity to impose that axiom stems from the fact that while it is trivially true in the classical world, it is not necessary so in our general setting.

A most interesting result is that the von Neumann-Lüders postulate which is central to Quantum Mechanics and informs about the impact of a measurement on the state of a system can be derived from a consistency requirement on choice behavior. When the belief-state (cognitive state) is updated according to the postulate, the agent conditional preferences reflect a single preference order. Of particular interest for behavioral economics is that in contrast with classical subjective expected utility theory, dynamic consistency of preferences does not entail the so-called recursive dynamic consistency. This is an expression of the fundamental distinction between the two settings namely that the resolution of uncertainty depends on the operation(s) performed to resolve it. We suggest that this very feature makes our theory an attractive candidate to develop "The Economics of Manipulation and Deception" called for by Akerlof and Shiller.

\section{References}

[1] Ahn D.S. and H. Ergin (2010) "Framing contingencies" Econometrica 78/2, 655-695.

[2] Alberti P.M. and A. Uhlmann (1982), Stochasticity and Partial Order, VEB Deutsch Verlag den Wissenchaften. 
[3] Anscombe F.J. and R.J. Aumann (1963) "A definition of Subjective Probability", The Annals of Mathematical Statictics, 34/1, 199-205.

[4] Bitbol M. ed. (2009), Physique Quantique et Sciences Humains, Edition CNRS Paris.

[5] Brandenburger A. and P. La Mura (2015) "Team Decision problems with Classical and Quantum Signals" Philosophical Transactions A, Royal Society December 2015, rsta.royalsocietypublishing.org 374:20150096

[6] Bruza P. and J. Busemeyer (2012)" Quantum Cognition and Decision-making" Cambridge University Press.

[7] Cohen M. and J.Y. Jaffray (1980), "Rational Behavior under Complete Ignorance" Econometrica 48/5, 1281-1299.

[8] Danilov V. I. and Lambert-Mogiliansky (2008) "Measurable System and Behavioral Sciences" Mathematical Social Sciences 55, 315-340.

[9] Danilov V. I. and A. Lambert-Mogiliansky (2010) "Expected Utility under Non-classical Uncertainty" Theory and Decision, 68 25-47.

[10] Deutsch D. (1999) " Quantum Theory of Probability and Decisions" Proceeding of the Royal Society, London A.455 3129-3137.

[11] Dubois F. and A. Lambert-Mogiliansky (2016) " Our (represented) world and quantumlike object" in Contextuality in Quantum Physics and Psychology, ed. Dzafarof et al, World Scientific, Advanced Series in Mathematical Psychology Vol. 6, 367-387.

[12] Fishburn, P.C. (1970), Utility Theory for Decision Making. Publications in Operations Research, No. 18. New York: John Wiley and Sons.

[13] Ghirardato P. (2002) "Revisisting Savage in a Conditionnal World" Economic Theory 20, 83-92.

[14] Gyntenberg J. and F. Hansen (2012), "Expected Utility with Subjective Events" Australian Journal of Mathematical Analysis and Applications 9/2, art.7, 1-21. 
[15] Kahneman D. and A. Tversky (2000) "Choice, Values and Frames" Cambridge University Press.

[16] Khrennikov, A., Haven, E. (2013). Quantum Social Science. Cambridge University Press.

[17] Lambert-Mogiliansky A., S. Zamir and H. Zwirn (2009) "Type-Indeterminacy a Model of the KT(Kahnemann and Tversky)Man", Journal of Mathematical Psychology vol $53 / 5,349-361$.

[18] Lehrer E. and E. Shmaya (2006) "A qualitative Approach to quantum probability" Proceedings of Royal Society 462, 2331-2344.

[19] Pitowsky I. (2003) "Betting on the outcome of measurements: a Bayesian theory of quantum probability"" Studies in History and Philosophy of Modern Physics, 34, 395414.

[20] Savage L. J. (1972) The Foundation of Statistics, Dover Publication, INC New York.

[21] Trueblod J. and J. Busemeyer (2012) " A Quantum Probability Model of Causal reasoning) Frontiers in Psychology Vol 3/138.

[22] Wang Z., T. Solloway, R. Shiffrin, and J. Busemeyer (2014) "Context Effect Produced by Question Order Reveals the Quantum Nature of Human Judgement" Psychological and Cognitive Sciences PNAS 1407756111.

\section{Appendix 1. Elementary facts about Hilbert spaces}

Hilbert space

Let $\mathbb{R}$ and $\mathbb{C}$ denote the fields of real and of complex numbers. For a complex number $z$, $\bar{z}$ denotes the complex conjugate number.

Definition 1. Let $H$ be a vector space over the field $\mathbb{C}$. An Hermitian form on $H$ is a mapping (.,. $): H \times H \rightarrow \mathbb{C}$ such that: a) it is linear in the first argument; b) $(v, w)=\overline{(w, v)}$ for any $v, w \in H$ (in particular, $(v, v)$ is a real number); c) $(v, v) \geq 0$ for any $v \in H$, and $(v, v)=0$ only for $v=0$. 
Vectors $v$ and $w$ are called orthogonal if $(v, w)=0$; in this case $(w, v)=0$ as well.

A Hilbert space is a vector space $H$ endowed with a Hermitian form, which is complete relatively to the norm $|v|=\sqrt{(v, v)}$. In order to avoid unnecessary difficulties and subtleties we assume further that $H$ has finite dimension; then $H$ automatically is complete.

When discussing lotteries and measurements we shall not be dealing so much with vectors in $H$ as with special operators (linear mappings from $H$ to $H$ ) called Hermitian operators.

\section{Hermitian operators}

Definition 2. A linear operator $A: H \rightarrow H$ is called Hermitian, if $(A v, w)=(v, A w)$ for any $v, w \in H$.

Clearly $(A v, v)$ is a real number for any $v \in H$. Hermitian operator $A$ is called nonnegative if $(A v, v) \geq 0$ for any $v$. For Hermitian operators $A$ and $B$ we write $A \geq B$ if $A-B$ is nonnegative. The identity operator $E(E v=v$ for every $v \in H)$ is Hermitian.

A most important, for the purpose of this work, class of Hermitian operator consists of projectors. A projector is an idempotent Hermitian operator, that is $P P=P$. Since $(P v, v)=(P P v, v)=(P v, P v) \geq 0$, any projector is nonnegative. Each projector $P$ define a vector subspace $V=\operatorname{Im} P \subset H$, consisting of vectors $v$ such that with $P v=v$. The kernel of the projector consists of vectors orthogonal to $V, \operatorname{Ker} P=V^{\perp}$. The set of projectors can be identify with the set of (closed) subspaces of $H$.

Any linear combination of Hermitian operators with real coefficients is an Hermitian operator. In other words, the set $\operatorname{Herm}(H)$ of Hermitian operators is a real vector space. The crucial importance of projectors is underlined by the following important theorem.

Spectral theorem. Let $A$ be a Hermitian operator. Then there exists a family of projectors $P_{i}$ and real numbers $a_{i}$ such that: 1) $P_{i} P_{j}=0$ for $i \neq j$, 2) $\sum_{i} P_{i}=E$, and 3) $A=\sum_{i} a_{i} P_{i}$

In other words, in some orthogonal basis the operator $A$ can be represented by a diagonal matrix (with real coefficients). The coefficients $a_{i}$ are eigenvalues of the operator $A$. The set of numbers $a_{i}$ is called the spectrum of the operator $A$. Clearly $A$ is nonnegative if and only if all coefficients $a_{i}$ are nonnegative. An operator $A$ is a projector if and only if its spectrum $\operatorname{Spec} A$ consists of 0 or 1 . 
Each nonnegative operator $A$ has a (unique) nonnegative square root $\sqrt{A}$ (or $A^{1 / 2}$, such that $(\sqrt{A})^{2}=A$ ). If $A=\sum_{i} a_{i} P_{i}$ is a spectral representation of $A$ then $\sqrt{A}=\sum_{i} \sqrt{a_{i}} P_{i}$.

Trace of operators

For arbitrary (not necessarily Hermitian) linear operator $A: H \rightarrow H$ it is possible to talk about its trace $\operatorname{Tr}(A)$. More precisely, for any quadratic matrix $A=\left(a_{i j}\right)$, the trace $\operatorname{Tr}(A)$ is defined as $\sum_{i} a_{i i}$, the sum of its diagonal elements. A remarkable property of the trace is its 'commutativity': $\operatorname{Tr}(A B)=\operatorname{Tr}(B A)$ for any quadratic matrix $A$ and $B$. This in particular implies that the trace of an operator is independent of the choice of basis, thereby allowing for an unambiguous definition of the trace of a linear operator.

For example, $\operatorname{Tr}(E)=\operatorname{dim} H$. More general, if $P$ is an (orthogonal) projector (on subspace $V=\operatorname{Im} P$ ) then $\operatorname{Tr}(P)=\operatorname{dim} V$. Due to the spectral theorem, we obtain that the trace of Hermitian operator $A=\sum a_{i} P_{i}$ is equal to $\sum a_{i} \mathrm{rk}\left(P_{i}\right)$ and, in particular, it is a real number. The trace of nonnegative operator $A$ is nonnegative and is strictly positive if $A \neq 0$.

For two Hermitian operators $A$ and $B$ define $(A, B)_{H e r m}=\operatorname{Tr}(A B)$. We assert that this 'scalar product' gives a structure of Euclidean space on the real vector space $\operatorname{Herm}(H)$. This follows from Lemma below.

Lemma. a) $(A, B)_{\text {Herm }}$ is a real number;

b) $(A, B)_{\text {Herm }}=(B, A)_{\text {Herm }}$;

c) $(A, A)_{\text {Herm }} \geq 0$ and is equal to 0 only if $A=0$.

Proof. Due to the 'commutativity' of the trace, $2 \operatorname{Tr}(A B)=\operatorname{Tr}(A B)+\operatorname{Tr}(B A)=$ $\operatorname{Tr}(A B+B A)$. It is easy to understand that the operator $A B+B A$ is Hermitian, hence its trace is real. This proves a).

b) follows from the 'commutativity' of the trace.

c) follows from the nonnegativity of the operator $A^{2}$.

\section{Appendix 2. Proof of Theorem 1}

Let $\preceq$ be a nice preference relation on the set $\mathrm{QL}(H)$. We shall be working with roulettevalued Q-lotteries, that is with expressions of the form $\sum_{i} l_{i} \otimes P_{i}$, where $\left(P_{i}, i \in I\right)$ is a 
PDU, and $\left(l_{i}, i \in I\right)$ is a collection of roulette lotteries on $X$. Let $\mathbf{Q L}_{\mathcal{P}}(H, \Delta(X))$ denote the set of all roulette valued Q-lotteries with base $\mathcal{P}$. We first provide a result that shows that mixtures of canonical Q-lotteries in $\mathbf{Q L}(H)$ and mixtures of Q-lotteries in $\mathbf{Q L}_{\mathcal{P}}(H, \Delta(X))$ are compatible.

Consider a lottery in the canonnical form $\sigma=\sum_{x} x \otimes P_{x}$, where $x \in X, P_{x}$ are nonnegative Hermitian operators which add up to $E, \sum_{x} P_{x}=E$. The mixture $\alpha \sigma+(1-\alpha) \tau$, where $\tau=\sum_{x} x \otimes Q_{x}$ and $\alpha \in[0,1]$, is given as $\sum_{x} x \otimes\left(\alpha P_{x}+(1-\alpha) Q_{x}\right)$. On the other hand a roulette valued Q-lottery writes $\sigma=\sum_{i} l_{i} \otimes P_{i}$, where $\mathcal{P}=\left(P_{i}, i \in I\right)$ is a measurement device (the base of the lottery) and $l_{i} \in \Delta(X)$. A mixture of such lotteries is defined by the following formula: $\alpha \sigma+(1-\alpha) \tau=\sum_{i}\left(\alpha l_{i}+(1-\alpha) r_{i}\right) P_{i}$, where $\tau=\sum_{i} r_{i} \otimes P_{i}$ is another Q-lottery in $\mathbf{Q L}_{\mathcal{P}}(H, \Delta(X))$ and $\alpha \in[0,1]$.

We next define the canonisation mapping can $: \mathbf{Q L}_{\mathcal{P}} \rightarrow \mathbf{Q L}_{c}$, which maps lottery $\sum_{i} l_{i} \otimes$ $P_{i}$ (where the roulette lottery $l_{i}$ has the form $\sum_{x} x \otimes l_{i}(x)$ i.e., $l_{i}$ gives value $x$ with probability $\left.l_{i}(x)\right)$ into the canonical lottery $\sum_{x} x \otimes\left(\sum_{i} l_{i}(x) P_{i}\right)$.

Lemma 2. The mapping can preserves the operation of mixture; that is, for any $\sigma, \tau \in \mathbf{Q L}_{\mathcal{P}}(H, \Delta(X))$ and $\alpha \in[0,1]$, $\operatorname{can}(\alpha \sigma+(1-\alpha) \tau)=\alpha \operatorname{can}(\sigma)+(1-\alpha) \operatorname{can}(\tau)$.

Proof. Assume we have two $\mathcal{P}$-based lotteries $\sigma=\sum_{i} l_{i} \otimes P_{i}$ and $\tau=\sum_{i} r_{i} \otimes P_{i}$ and some $\alpha \in[0,1]$. We want to show that $\operatorname{can}(\alpha \sigma+(1-\alpha) \tau)=\alpha \operatorname{can}(\sigma)+(1-\alpha) \operatorname{can}(\tau)$.

The left hand side is equal to $\operatorname{can}\left(\sum_{i}\left(\alpha l_{i}+(1-\alpha) r_{i}\right) \otimes P_{i}\right)=$

$\sum_{x} x \otimes \sum_{i}\left(\alpha l_{i}+(1-\alpha) r_{i}\right)(x) P_{i}=\sum_{x} x \otimes \sum_{i}\left(\alpha l_{i}(x)+(1-\alpha) r_{i}(x)\right) P_{i}=$

$=\sum_{x} x \otimes \sum_{i}\left(\alpha \sum_{i} l_{i}(x)+(1-\alpha) \sum_{i} r_{i}(x)\right) P_{i}$.

The right hand side is $\alpha \sum_{x} x \otimes\left(\sum_{i} l_{i}(x) P_{i}\right)+(1-\alpha)\left(\sum_{x} x \otimes \sum_{i} r_{i}(x) P_{i}\right)$ $=\alpha \operatorname{can}(\sigma)+(1-\alpha) \operatorname{can}(\tau)$.

Returning to the proof of Theorem 1 , let $\preceq_{\Delta}$ denote the derived preference relation on the set $\Delta(X)$ of roulette lotteries. The assertion of the theorem is true if the preference $\preceq$ is trivial. Indeed, we can take $u$ to be a constant and take an arbitrary functional $\beta$. So, from now on, we assume that the preference $\preceq$ is nontrivial. That is $\tau \prec \sigma$ for some Q-lotteries $\sigma=\sum_{i} l_{i} \otimes P_{i}$ and $\tau=\sum_{j} m_{j} \otimes Q_{j}$

Claim 0. Let $l^{*}$ be the best lottery among $\left(l_{i}\right)$ with respect to the derived weak order $\preceq_{\Delta}$. Then $\sigma \preceq l^{*} \otimes E$. 
Proof. Consider the 'constant' lottery $\sigma^{*}=\sum_{i} l^{*} \otimes P_{i}$. Due to A4, $\sigma \preceq \sigma^{*}$. Due to A0, $\sigma^{*} \approx l^{*} \otimes E$. Due to transitivity of $\preceq$ (see A1) we conclude that $\sigma \preceq l^{*} \otimes E$.

Corollary 1. The derived preference $\preceq_{\Delta}$ is non-trivial, that is $l_{*} \prec_{\Delta} l^{*}$ for some ordinary lotteries $l_{*}$ and $l^{*}$.

Proof. Indeed, if $m_{*}$ ia the worst lottery among $\left(m_{j}\right)$, then we have

$$
m_{*} \otimes E \preceq \tau \prec \sigma \preceq l^{*} \otimes E,
$$

whence $m_{*} \prec_{\Delta} l^{*}$.

We fix such lotteries $l_{*} \prec_{\Delta} l^{*}$; a function $u: \Delta(X) \rightarrow \mathbb{R}$ is said to be normalized if $u\left(l_{*}\right)=0$ and $u\left(l^{*}\right)=1$.

Fix now some measurement device $\mathcal{P}=\left(P_{i}, i \in I\right)$, and let $\mathbf{Q L}_{\mathcal{P}}(H, \Delta(X))$ denote the set of all roulette valued Q-lotteries with base $\mathcal{P}$. We first extend $\preceq$ into a preference relation $\preceq_{\mathcal{P}}$ defined on $\mathbf{Q L}_{\mathcal{P}}(H, \Delta(X))$ by setting $\sigma \preceq \tau$ if and only if $\operatorname{can}(\sigma) \preceq \operatorname{can}(\tau)$ for any $\sigma, \tau \in \mathbf{Q L}_{\mathcal{P}}(H, \Delta(X))$.

Claim 1. There exists a normalized affine function $u_{\mathcal{P}}$ on $\Delta(X)$ and a function $\beta_{\mathcal{P}}$ on the set of outcomes $I\left(\beta_{\mathcal{P}}(i) \geq 0\right.$ and $\left.\sum_{i} \beta_{\mathcal{P}}(i)=1\right)$ such that the preference $\preceq_{\mathcal{P}}$ is represented by the function $U_{\mathcal{P}}, U_{\mathcal{P}}\left(\sum_{i} l_{i} \otimes P_{i}\right)=\sum_{i} u_{\mathcal{P}}\left(l_{i}\right) \beta_{\mathcal{P}}(i)$. Moreover, both $u_{\mathcal{P}}$ and $\beta_{\mathcal{P}}$ are unique.

Proof. Each Q-lottery $\sigma=\sum_{i} l_{i} \otimes P_{i}$ can be considered as a 'horse' lottery $f: I \rightarrow \Delta(X)$, where $f(i)=l_{i}$. Moreover, due to axioms A1-A4 and Lemma 2, the relation $\preceq_{\mathcal{P}}$ satisfies all the Anscombe-Aumann axioms. Therefore, by theorem 13.2 in Fishburn (1970), we obtain an affine utility function $u_{\mathcal{P}}$ on $\Delta(X)$ and a probability measure $\beta_{\mathcal{P}} \in \Delta(I)$ that achive the representation stated in Claim 1. The uniqueness of $\beta_{\mathcal{P}}$ is also given by this theorem. The uniqueness of $u_{\mathcal{P}}$ follows from normalization of $u_{\mathcal{P}}$.

Claim 2. The functions $u_{\mathcal{P}}$ are independent of $\mathcal{P}$ (and we denote them as u).

Proof. Due to A0, each of the functions $u_{\mathcal{P}}$ represents the derived preference $\preceq_{\Delta}$ on $\Delta(X)$. Therefore they are positive affine transformations of each other. Normalization gives that they are in fact equal to each other. 
Claim 3. For any Q-lottery $\sigma$, there exists an ordinary lottery $l \in \Delta(X)$ such that $\sigma$ is equivalent to $l$, that is $\sigma \approx l \otimes E$.

Proof. Let $\sigma=\sum_{i} l_{i} \otimes P_{i}$, and $l^{b}$ (correspondingly, $l^{w}$ ) is a best (corresp., a worst) lotteries among $\left(l_{i}, i \in I\right)$. Due to A4, we have $\sum_{i} l^{w} \otimes P_{i} \preceq \sigma \preceq \sum_{i} l^{b} \otimes P_{i}$, and all these Q-lotteries have the same base $\mathcal{P}=\left(P_{i}, i \in I\right)$. Therefore we can apply Claim 1 , which gives inequalities

$$
u\left(l^{w}\right) \leq \sum_{i} u\left(l_{i}\right) \beta_{\mathcal{P}}(i) \leq u\left(l^{b}\right)
$$

Hence $\sum_{i} u\left(l_{i}\right) \beta_{\mathcal{P}}(i)=\alpha u\left(l^{w}\right)+(1-\alpha) u\left(l^{b}\right)=u(l)$ for some $\alpha \in[0,1]$, where $l=\alpha l^{w}+(1-$ $\alpha) l^{b}$. By Claim 1, we have $\sigma \approx \sum_{i} l \otimes P_{i} \approx l \otimes E$.

Due to Claim 1, the function $U_{\mathcal{P}}$ allows to compare Q-lotteries with base $\mathcal{P}$. But we assert that it allows to compare Q-lotteries with different bases as well.

Claim 4. Let $\sigma=\sum_{i} l_{i} \otimes P_{i}$ be a Q-lottery with a base $\mathcal{P}=\left(P_{i}, i \in I\right)$, and let $\tau=\sum_{j} m_{j} \otimes Q_{j}$ be a Q-lottery with a base $\mathcal{Q}=\left(Q_{j}, j \in J\right)$. Then $\sigma \preceq \tau$ if and only if $U_{\mathcal{P}}(\sigma) \leq U_{\mathcal{Q}}(\tau)$.

Proof. Due to Claim 3, the lottery $\sigma$ is equivalent to some lottery $l \otimes E$, or to the lottery $\sum_{i} l \otimes P_{i}$. Therefore, $U_{\mathcal{P}}(\sigma)=U_{\mathcal{P}}\left(\sum_{i} l \otimes P_{i}\right)=u(l)$. Similarly, $\tau$ is equivalent to $m \otimes E$, and $U_{\mathcal{Q}}(\tau)=u(m)$. Now

$$
\sigma \preceq \tau \Leftrightarrow l \otimes E \preceq m \otimes E \Leftrightarrow U_{\mathcal{P}}(\sigma)=u(l) \leq u(m)=U_{\mathcal{Q}}(\tau) .
$$

Let us return now to the functions $\beta_{\mathcal{P}}$. We assert that $\beta_{\mathcal{P}}(i)$ depends only on the operator $P_{i}$, not of $\mathcal{P}$ and $i$.

Claim 5. Let $\mathcal{P}=\left(P_{1}, \ldots, P_{n}\right)$ and $Q=\left(Q_{1}, \ldots Q_{k}\right)$ be two measurement devices (bases), and $P_{1}=Q_{1}=R$. Then $\beta_{\mathcal{P}}(1)=\beta_{\mathcal{Q}}(1)$.

Proof. Consider Q-lottery $\sigma=l^{*} \otimes P_{1}+\sum_{i=2}^{n} l_{*} \otimes P_{i}$ with base $\mathcal{P}$. Its $\mathcal{P}$-utility $U_{\mathcal{P}}(\sigma)$ is equal to $\beta_{\mathcal{P}}(1)$. Now let us form the auxiliary base $\mathcal{R}=(R, E-R)$ and the following Q-lottery $\rho=l^{*} \otimes R+l_{*}(E-R)$. Since $E-R=P_{2}+\ldots+P_{n}$, the lottery $\rho$ is equivalent to $\sigma$ (see Axiom A0). Therefore $\mathcal{R}$-utility $U_{\mathcal{R}}(\rho)$ (which equals $\beta_{\mathcal{R}}(1)$ ) is, by Claim 4 , equal to $\beta_{\mathcal{P}}(1)$. The same applies to $\mathcal{Q}$ and gives the equality $\beta_{\mathcal{R}}(1)=\beta_{\mathcal{Q}}(1)$. Together with the 
equality $\beta_{\mathcal{R}}(1)=\beta_{\mathcal{P}}(1)$ we obtain the equality $\beta_{\mathcal{P}}(1)=\beta_{\mathcal{Q}}(1)$.

As a consequence, we can speak about the number $\beta(P)$ for any 'event' $P$, that is for any hermitian operator $P, 0 \leq P \leq E . \quad \beta(P)$ is $\mathcal{R}$-utility $U_{\mathcal{R}}$ of the following Q-lottery $l^{*} \otimes P+l_{*} \otimes(E-P)$. Correspondingly, the utility of an arbitrary Q-lottery $\sigma=\sum_{i} l_{i} \otimes P_{i}$ can be rewritten as

$$
U(\sigma)=\sum_{i} u\left(l_{i}\right) \beta\left(P_{i}\right) .
$$

Obviously, $\beta(0)=0$ and $\beta(E)=1$. Moreover, $\beta(P) \geq 0$ for any 'event' $P$, and $\sum_{i} \beta\left(P_{i}\right)=1$ provided $\sum_{i} P_{i}=E$.

Claim 6. If $0 \leq P, 0 \leq Q$, and $P+Q \leq E$, then $\beta(P+Q)=\beta(P)+\beta(Q)$.

Proof. Indeed, consider the Q-lottery $l^{*} \otimes P+l^{*} \otimes Q+l_{*} \otimes(E-P-Q)$. Its utility is $\beta(P)+\beta(Q)$. On the other hand, due to $\mathrm{A} 0$, this lottery is equivalent to the lottery $l^{*} \otimes(P+Q)+l_{*} \otimes(E-P-Q)$, whose utility is $\beta(P+Q)$.

Claim 6 implies that $\beta$ can be extended to a (unique) linear functional $\beta$ on the vector space $\operatorname{Herm}(H)$. Obviously, $\beta(A) \geq 0$ for $A \geq 0$, and $\beta(E)=1$. That is $\beta$ is a belief functional. This completes the proof of Theorem 1.

\section{Appendix 3. Proof of Theorem 2}

Proof of Assertion a). Here we can work with lotteries in the form of Hermitian operators. Utility $U(A)$ of such an operator $A$ is equal to $\operatorname{Tr}(A B)$ and utility under the condition $P$ is equal to $U(A \mid P)=\operatorname{Tr}(A P B P)$ (up to the factor $\operatorname{Tr}(P B P)$ ).

Proving property A5. We assume that $A \mid W=0$ and have to show that $U(A \mid P)=0$. Note that $A \mid P=0$ is equivalent to $P A P=0$. Now $U(A \mid P)=\operatorname{Tr}(A P B P)=\operatorname{Tr}(P A P B)=$ $\operatorname{Tr}(0 B)=0$.

Proving property A6. Here we assume that $A$ commute with $P$ and that $(E-P) A=0$ (that is $A=P A=A P)$. We have to show that $U(A \mid P)$ is equal to $U(A)$. But $U(A \mid P)=$ $\operatorname{Tr}(A P B P)=\operatorname{Tr}(P A P B)=\operatorname{Tr}(A B)=U(A)$, because $P A P=A$.

Proof of Assertion b). To proof it, we define (for an arbitrary Q-lottery $\sigma=\sum_{i} x_{i} \otimes P_{i}$ and an event $W$ given by a projector $P$ ) some special (adapted to $P$ ) Q-lottery that we 
denote $a d_{W}(\sigma)$. Roughly speaking, $a d_{W}(\sigma)=\sum_{i} x_{i} \otimes P P_{i} P$. However, the sum $\sum_{i} P P_{i} P$ is equal to $P$, not to $E$. Therefore we add to this sum a tail-end $x_{*} \otimes(E-P)$. Here $x_{*}$ is a prize with zero utility. The final formula yields

$$
a d_{W}(\sigma)=\sum_{i} x_{i} \otimes P P_{i} P+x_{*} \otimes(E-P)
$$

Lemma 3. a) $\sigma\left|W=a d_{W}(\sigma)\right| W$;

b) the adapted lottery $\operatorname{ad}_{W}(\sigma)$ is compatible with $P$;

c) for any Q-lottery $\sigma$ we have $a d_{W}(\sigma) \mid W^{\perp}=x_{*} \otimes(E-P)$.

Proof. a) It is obvious that $P_{i}\left|W=P P_{i} P\right| W$. Moreover, $(E-P) \mid W=P(E-P)=0$.

b) For any $i$ we have $P P P_{i} P=P P_{i} P P$, since $P P=P$. Moreover, $P(E-P)=$ $(E-P) P=0$.

c) It is clear that $(E-P) P P_{i} P=0$ for any $i$.

Proposition 4. Suppose that a preference relation $\preceq_{W}$ on the set $\mathrm{QL}(H)$ is a weak order and satisfies the axioms A5 and A6. Then it is given by the following explicit formula (where $\sigma$ and $\tau$ are $Q$-lotteries on $H)$ :

$$
\sigma \preceq_{W} \tau \text { if and only if } a d_{W}(\sigma) \preceq a d_{W}(\tau)
$$

Indeed, due to the axiom A5 and Lemma 3, we have $\sigma \approx_{W} a d_{W}(\sigma)$ and $\tau \approx_{W} a d_{W}(\tau)$. Applying axiom A6 to the lotteries $a d_{W}(\sigma)$ and $a d_{W}(\tau)$ (which is possible due to points 2) and 3) of Lemma 3), we obtain that $a d_{W}(\sigma) \preceq a d_{W}(\tau)$ if and only if $a d_{W}(\sigma) \preceq{ }_{W} a d_{W}(\tau)$. The rest follows from the transitivity of $\preceq_{W}$.

There remains to recall that (unconditional) utility of lottery $a d_{W}(\sigma)$ is equal to $\operatorname{Tr}\left(\operatorname{Sh}\left(a d_{W}(\sigma)\right) B\right)$. If $A=S h(\sigma)$ then $S h\left(a d_{W}(\sigma)\right)=P A P+0(E-P)=P A P$. Therefore $U\left(a d_{W}(\sigma)\right)=$ $\operatorname{Tr}(P A P B)=\operatorname{Tr}(A P B P)=\operatorname{Tr}(P B P) \operatorname{Tr}\left(A B^{u p}\right)$, what is (up to the factor $\operatorname{Tr}(P B P)$ ) the utility of $\sigma$ with respect to 'updated' belief operator $B^{u p}=P B P / \operatorname{Tr}(P B P)$. This completes the proof of Theorem 2. 


\section{Appendix 4. Proof of Proposition 1.}

We here prove an assertion that generalizes Propostion 1 as we substitute projector $P$ with an arbitrary 'fuzzy-projector' $F$, that is an operator $0 \leq F \leq E$ (see Remark 4 of Section 4). The posterior $B^{u p}$ is given as $\sqrt{F} B \sqrt{F} / \operatorname{Tr}(\sqrt{F} B \sqrt{F})=\sqrt{F} B \sqrt{F} / \operatorname{Tr}(F B)$. We shall denote it as $B \mid F$.

Proposition 1'. The distance between a prior $B$ and the posterior $B \mid F$ is $O((1-$ $\left.\operatorname{Tr}(F B))^{1 / 4}\right)$.

In other words, if $\varepsilon=1-\operatorname{Tr}(F B)$ then the distance between $B$ and $B \mid F$ is $O\left(\varepsilon^{1 / 4}\right)$.

Proof. Choose an orthonormal basis of $H$ in which the operator $F$ (as well as $\sqrt{F}$ ) has diagonal form

$$
F=\operatorname{diag}\left(f_{1}, \ldots, f_{n}\right)
$$

In this basis operator $B$ is represented by Hermitian matrix $\left(b_{i j}\right)$, where $i$ and $j$ run over 1 to $n=\operatorname{dim} H$. The matrix $\sqrt{F} B \sqrt{F}$ has coefficients $\sqrt{f_{i}} b_{i j} \sqrt{f_{j}}$. The matrix $\sqrt{F} B \sqrt{F}$ differs of $B \mid F=\sqrt{F} B \sqrt{F} / \operatorname{Tr}(F B)$ by less than $O(\varepsilon)$. Thus we need to compare $B$ and $\sqrt{F} B \sqrt{F}$ and to show that the distance between them is $O\left(\varepsilon^{1 / 4}\right)$. Or equivalenetly we need to show that (for any $i$ and $j$ ) distance between $b_{i j}$ and $\sqrt{f_{i}} b_{i j} \sqrt{f_{j}}$ is $O\left(\varepsilon^{1 / 4}\right)$.

Let us divide the set of indices $i$ from $\{1, \ldots, n\}$ into two groups. Say that an index $i$ is non-essential, if $b_{i i} \leq \sqrt{\varepsilon}$, and is essential in the opposite case.

Lemma 4. If $i$ is non-essential then $\left|b_{i j}\right| \leq \varepsilon^{1 / 4}$.

Proof. A sub-matrix of the matrix $B$, formed by the rows and columns from the set $\{i, j\}$, is Hermitian and therefore has non-negative determinant $b_{i i} b_{j j}-\left|b_{i j}\right|^{2}$. That is $\sqrt{\varepsilon} \geq$ $b_{i i} b_{i j} \geq\left|b_{i j}\right|^{2}$.

Due to Lemma A, if $i$ or $j$ is non-essential then the coefficient $b_{i j}$ of the matrix $B$ as well as the corresponding coefficient $b_{i j} \sqrt{f_{i}} \sqrt{f_{j}}$ of the matrix $\sqrt{F} B \sqrt{F}$ is $\leq \varepsilon^{1 / 4}$. Therefore the distance between them is $\leq 2 \varepsilon^{1 / 4}$.

Hence we can suppose that the both indexes $i$ and $j$ are essential.

Lemma 5. If an index $i$ is essential then $f_{i}=1+O\left(\varepsilon^{1 / 2}\right)$. 
Proof. We have $\operatorname{Tr}(F B)=1-\varepsilon$, that is $\sum_{i} f_{i} b_{i i}=1-\varepsilon$. Moreover the trace of $B$ is equal to 1 , that is $\sum_{i} b_{i i}=1$. Subtracting we obtain that $\sum_{i} b_{i i}\left(1-f_{i}\right)=\varepsilon$, therefore each term of this sum is less or equal to $\varepsilon, b_{i i}\left(1-f_{i}\right) \leq \varepsilon$. . In particular, if $i$ is essential then $b_{i i}>\varepsilon^{1 / 2}$ and $1-f_{i}<\varepsilon^{1 / 2}$, that is $f_{i}=1+O\left(\varepsilon^{1 / 2}\right)$.

As a corollary we obtain (for an essential $i$ ) that $\sqrt{f_{i}}=1+O\left(\varepsilon^{1 / 2}\right)$.

Let us return to evaluation of the distance between $b_{i j}$ and $b_{i j} \sqrt{f_{i}} \sqrt{f_{j}}$ in the case when $i$ and $j$ are essential indexes. It is clear that $b_{i j}-b_{i j} \sqrt{f_{i}} \sqrt{f_{j}}=b_{i j}\left(1-\left(1+O\left(\varepsilon^{1 / 2}\right)\right)\left(1+O\left(\varepsilon^{1 / 2}\right)\right)\right)$. The module of this number is no more than $\left|b_{i j}\right|$ (which is $\leq 1$ ) multiplied by $Q\left(\varepsilon^{1 / 2}\right)$. Therefore the distance is no more than $\leq O\left(\varepsilon^{1 / 2}\right) \leq O\left(\varepsilon^{1 / 4}\right)$ which proves Proposition $1^{6}$. 